\title{
Simulating school closure policies for cost effective pandemic decision making
}

\author{
Ozgur M Araz ${ }^{1 *}$, Paul Damien², David A Paltiel ${ }^{3}$, Sean Burke ${ }^{4}$, Bryce van de Geijn ${ }^{4}$, Alison Galvani3 ${ }^{3,5}$ and \\ Lauren Ancel Meyers ${ }^{4,6}$
}

\begin{abstract}
Background: Around the globe, school closures were used sporadically to mitigate the 2009 H1N1 influenza pandemic. However, such closures can detrimentally impact economic and social life.

Methods: Here, we couple a decision analytic approach with a mathematical model of influenza transmission to estimate the impact of school closures in terms of epidemiological and cost effectiveness. Our method assumes that the transmissibility and the severity of the disease are uncertain, and evaluates several closure and reopening strategies that cover a range of thresholds in school-aged prevalence (SAP) and closure durations.

Results: Assuming a willingness to pay per quality adjusted life-year (QALY) threshold equal to the US per capita $\operatorname{GDP}(\$ 46,000)$, we found that the cost effectiveness of these strategies is highly dependent on the severity and on a willingness to pay per QALY. For severe pandemics, the preferred strategy couples the earliest closure trigger ( $0.5 \%$ SAP) with the longest duration closure (24 weeks) considered. For milder pandemics, the preferred strategies also involve the earliest closure trigger, but are shorter duration (12 weeks for low transmission rates and variable length for high transmission rates).
\end{abstract}

Conclusions: These findings highlight the importance of obtaining early estimates of pandemic severity and provide guidance to public health decision-makers for effectively tailoring school closures strategies in response to a newly emergent influenza pandemic.

\section{Background}

Influenza pandemics affect millions of people worldwide, exacting significant costs on the global economy in terms of illness, deaths, medical resources, and loss of productivity. In 2009, the influenza A (H1N1) pandemic challenged the world's socio-economic system and forced public health agencies to take aggressive measures to mitigate its social and economic impact in some parts of the world. It demonstrated the importance of non-pharmaceutical interventions prior to the distribution of an effective vaccine [1]. However, during the early emergence of a new influenza pandemic, estimates of the transmissibility and severity (i.e. case fatality rate) of the new virus are typically based on noisy and sparse data [2]. Public health officials thus face considerable uncertainties when making decisions about early stage pandemic mitigation strategies.

\footnotetext{
* Correspondence: ozgur.araz@unmc.edu

'College of Public Health, University of Nebraska Medical Center, Omaha, NE 68198, USA

Full list of author information is available at the end of the article
}

School closures offer a radical form of social distancing with great potential to slow the early growth of a pandemic, at significant economic and social costs [3, 4]. Because of this trade-off, knowing the transmissibility and severity of a spreading pandemic are particularly critical to effective school closure policy-making. During the 2009 H1N1 pandemic, schools closures were sporadic in the US, triggered by diverse decision-making entities under variable situations $[5,6]$ and other countries, e.g. Mexico, implemented nationwide school closures that lasted for about 2 weeks.

In developing school closure policies for future pandemics, researchers and public health officials have used models and quantitative analyses to evaluate their efficacies and costs under possible pandemic scenarios [3, 7]. A number of studies have quantified the impact of school closures on cumulative attack rates, but have not evaluated the social and economic costs of such policies $[6,8-$ 12]. Some of these studies demonstrate school closures can have a significant impact on the basic reproduction 
number and on the overall spread of disease $[4-7,10,13$, 14]. Other studies have estimated the direct economic cost of influenza and school closures and their indirect impacts on the health care system [3, 4, 15-20]. One advocates, for example, 26 weeks of school closure in conjunction with other policies [4]. For other modes of intervention, a number of studies simultaneously assess epidemiological and economic impacts using stochastic agent-based models of pandemic influenza transmission and decisionanalytic approaches $[4,12,20-22]$. While a few of these consider one or very few school closure options among a larger spectrum of intervention measures $[4,12,23]$, none systematically explore the cost effectiveness and epidemiological impact of a large set of school closure policies nor assess the political, social or economic viability of such policies for mild pandemics such as $2009 \mathrm{H} 1 \mathrm{~N} 1$ [24].

Here, we combine a decision analytic approach with economic and mathematical infectious disease modeling to estimate the impact of school closures in terms of both epidemiological and cost effectiveness. We consider several scenarios for the transmissibility and severity of the disease, and evaluate diverse closure and reopening strategies covering a range of thresholds in school-aged prevalence (SAP) and closure durations.

Table 1 Model parameters

\begin{tabular}{|c|c|c|c|c|c|c|c|c|}
\hline \multicolumn{8}{|c|}{ Sociological Parameters } & \multirow[t]{2}{*}{ Reference(s) } \\
\hline \multicolumn{4}{|l|}{ Age Groups } & \multicolumn{4}{|c|}{ Population } & \\
\hline \multicolumn{4}{|l|}{$<=5$} & \multicolumn{4}{|c|}{$2,019,138$} & FedStat [29] \\
\hline \multicolumn{4}{|l|}{$>5<=18$} & \multicolumn{4}{|c|}{$4,655,105$} & \\
\hline \multicolumn{4}{|l|}{$>=19$} & \multicolumn{4}{|c|}{$17,612,731$} & \\
\hline \multicolumn{4}{|l|}{ Total } & \multicolumn{4}{|c|}{$24,326,974$} & \\
\hline \multicolumn{4}{|c|}{$\%$ With Children $<16$} & \multicolumn{4}{|l|}{53.58} & FedStat [29] \\
\hline \multicolumn{4}{|c|}{ Average Daily Salary (\$/day) } & \multicolumn{4}{|c|}{135.25} & \\
\hline \multicolumn{4}{|c|}{ Employment Rate (\%) } & \multicolumn{4}{|l|}{93} & \\
\hline \multicolumn{4}{|c|}{ Single Parents (\%) } & \multicolumn{4}{|l|}{20} & Lempel et al. [3] \\
\hline \multicolumn{4}{|c|}{ Working parents missing work (\%) } & \multicolumn{4}{|l|}{14} & \\
\hline \multicolumn{4}{|c|}{ Days Work Missed } & \multicolumn{4}{|c|}{2.5 (Couples), 5 (Single Parents) } & \\
\hline \multicolumn{4}{|c|}{ Discount Rate (\%) } & \multicolumn{4}{|l|}{3} & {$[4,26]$} \\
\hline \multicolumn{4}{|c|}{ Cost of Monitoring Infections (\$) } & \multicolumn{4}{|l|}{100} & Assumed \\
\hline Contact Rates & le/day) & & & & & & & Mossong et al. [30] \\
\hline Schools open & & Adults & & & Childrer & & & \\
\hline Adults & & 7 & & & 1 & & & \\
\hline Children & & 6 & & & 10 & & & \\
\hline Schools close & & Adults & & & Childres & & & \\
\hline Adults & & 7 & & & 1 & & & \\
\hline Children & & 4 & & & 2 & & & \\
\hline Transmission & & Adults & & & Childr & & & Cauchemez et al. [8] \\
\hline & & Min & Most Likely & Max & Min & Most Likely & Max & \\
\hline $\mathbf{R}_{\mathbf{0}} \sim[1.1-1.5]$ & Adults & 0.123 & 0.145 & 0.168 & 0.141 & 0.167 & 0.193 & \\
\hline & Children & 0.227 & 0.269 & 0.31 & 0.262 & 0.309 & 0.257 & \\
\hline $\mathbf{R}_{\mathbf{0}} \sim[1.5-2.1]$ & Adults & 0.178 & 0.201 & 0.234 & 0.205 & 0.231 & 0.269 & \\
\hline & Children & 0.33 & 0.372 & 0.434 & 0.38 & 0.427 & 0.499 & \\
\hline Other Influen & Imeters & & & & & & & \\
\hline Latent period & & 3 days & & & & & & Gojovic et al. [12] \\
\hline Infectious per & & 6 days & & & & & & \\
\hline Case Fatality & CFR) & Adults & & & Childrer & & & \\
\hline Low Severity Sce & & $0.159 \%$ & & & $0.01 \%$ & & & Presanis et al. [31] \\
\hline High Severity Sc & & $2 \%$ & & & $2 \%$ & & & Chowell et al. [32] \\
\hline
\end{tabular}




\section{Methods}

We evaluate a range of pandemic influenza school closure and reopening strategies, under four different scenarios for the transmissibility and severity of the pandemic. The impacts of school closures on influenza attack rates are estimated using an age-specific mass action model, i.e., all individuals act similarly but separately from each other in a homogenously mixed population [25]. Each school closure policy consists of a prevalence-based closure trigger and either a prevalence-based or fixed duration reopening trigger. Our cost effectiveness analysis followed the recommendations of the Panel on Cost-Effectiveness in Health and Medicine [26], and considered a detailed model of the societal costs of school closures as outlined by Muenning [27].

\section{Model}

We use an age-structured SEIR (Susceptible-Exposed-Infectious-Recovered) model [28] of pandemic influenza transmission, based on population data for the state of Texas from FedStat [29] (see Table 1). Infected individuals are assumed to enter a latent period (with average duration of three days) during which they are symptomfree, followed by an infectious period (with average duration of six days) during which they are both symptomatic and infectious [12] See Additional file 1 for one-way sensitivity analyses on both latent and infectious periods.

We considered two levels of pandemic severity: relatively low case fatality rates (CFR) of $0.01 \%$ for the school age population and $0.16 \%$ for the adult population, based on estimates for the 2009 (H1N1) pandemic [31] and higher CFR's ( $2 \%$ for both of the age groups), as estimated for the 1918 pandemic [32].

On the other hand, estimates for the basic reproduction number $\left(R_{0}\right)$ of $2009 \mathrm{H} 1 \mathrm{~N} 1$ pandemic influenza (and other strains of pandemic flu) range between 1.1 and 2.1 [33]. Accordingly, we considered two transmission rate scenarios: $R_{0}$ values are sampled from age-specific triangular distributions with values ranging either from 1.1 to 1.5 (low transmission scenario) with mean 1.3 or from 1.5 to 2.1 (high transmission scenario) with mean 1.8 (Table 1). Triangular distributions were chosen because of a lack of consensus about the distribution of reproduction numbers for pandemic influenza; they have only three relatively tangible parameters (minimum, maximum, and most likely value) that are readily subjected to sensitivity analyses across likely ranges.

Mixing rates between age groups in the model were estimated from the contact numbers reported in Mossong et al. [30] (Table 1). For estimates of mixing rates during school closures, contacts taking place in schools were excluded, i.e. there is no compensatory contact included in the model during school closures. Age-specific transmission rates were then calculated based on the mixing rates and $R_{0}$ (see Additional file 1 and Table 1 ).

The mathematical formulation of the age-structured SEIR model is given in Additional file 1. Numerical simulations were initiated with five exposed individuals per 1000 in the school-aged population. For each combination of epidemic scenario (high/low transmission with high/low severity) and school closure policy option, we ran 200 simulations, each based on a random sample of $R_{0}$, i.e. sampling values from the corresponding probability distributions (Table 1).

\section{School closure policies and the decision pathway}

The possible closure triggers were a range of SAP thresholds (prevalence in children ages 5-18), based on the assumption that prevalence in this age group can be

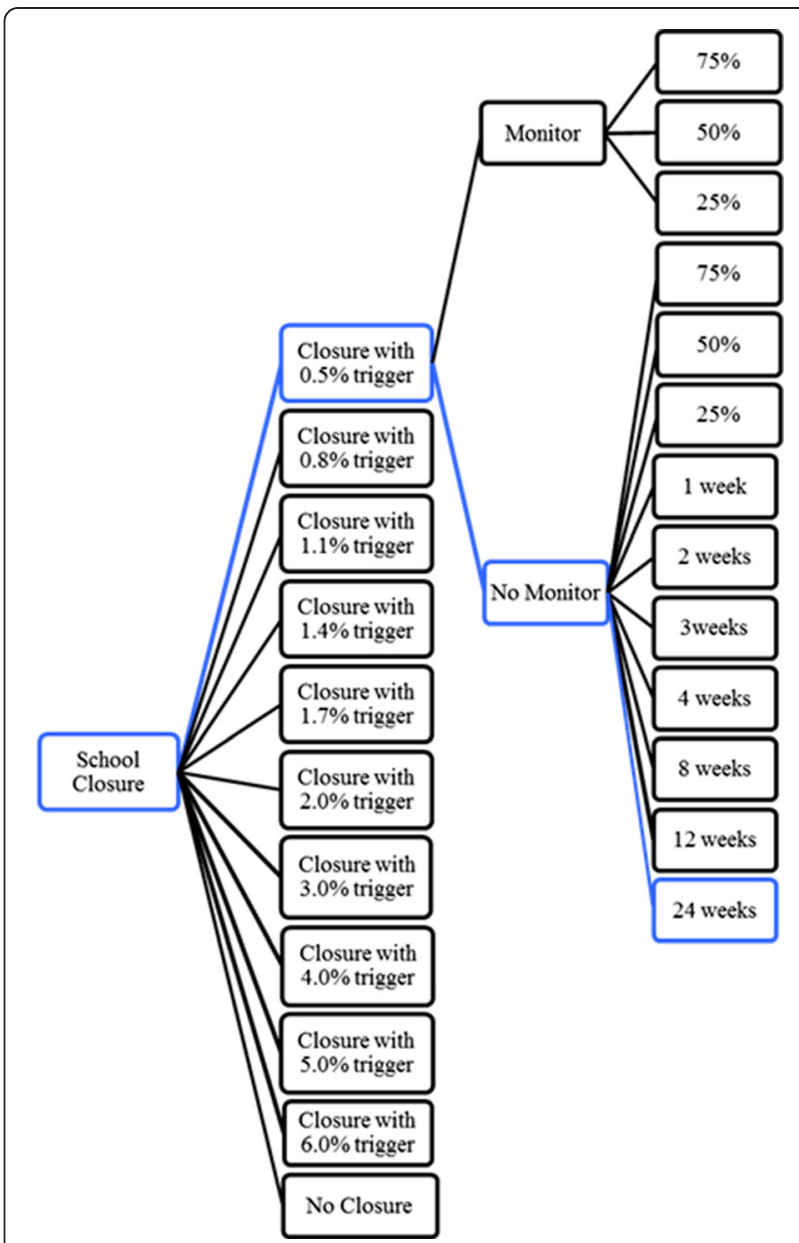

Figure 1 School closure policy pathway for high transmission scenarios. The full tree for the $0.5 \%$ school-age prevalence closure trigger is shown. All other closure triggers have the same decision options as the $0.5 \%$ trigger, but are not depicted. The policy option in blue (24-week closure triggered by $0.5 \%$ school-aged prevalence) is one of the efficient options in the tree under both high transmission scenarios (high and low severity). 
estimated in real-time from school absenteeism data. For the low transmission scenario, the SAP triggers were $0.5 \%, 0.8 \%, 1.1 \%, 1.4 \%, 1.7 \%$ and $2.0 \%$. For the high transmission scenario, the SAP triggers included these options plus $3 \%, 4 \%, 5 \%$ and $6 \%$. These were constrained by the peak prevalence predicted by the model $(2.2 \%$ and $7.9 \%$ for the school age population with a peak after 113 and 71 days in the low and high transmission scenarios, respectively). The ten reopening policy options were fixed duration closures of $1,2,3,4,8,12$, and 24 weeks and declines in SAP to $75 \%, 50 \%$ or $25 \%$ of that measured at the time of school closure. Policies that reopen schools based on drops in SAP depend on continual epidemiological surveillance, which can be costly and unreliable. Our model assumes that existing surveillance systems allow one-week delayed estimates of SAP, and that additional monitoring would allow real-time estimates of SAP, at a cost. In both cases, we assume that the SAP estimates are exact.

Fixed duration options may be more practical than prevalence-based re-openings, because fixed durations allow public health agencies, schools and families to plan in advance. However, we do not explicitly model any additional inconvenience or costs associated with trigger-based re-openings and the study is limited in its ability to capture the magnitude of how rational individuals will adapt economic behavior to minimize costs.

Figure 1 illustrates a small portion of the policy tree for the high transmission scenarios. Every school closure policy option is represented by a unique path from the left to the right through the tree. For example, the policy following the top-most branches is a school closure that (a) is triggered to close when SAP reaches $0.5 \%$, (b) institutes additional monitoring for changes in SAP during the closure, and (c) is triggered to re-open when the SAP drops to $25 \%$ of that observed at the time of the initial closure.

\section{Cost calculation and cost effectiveness analysis}

We estimated the societal costs and health outcomes for each policy and for a base case of no school closure during the first 180 days of the pandemic (prior to the availability of vaccines).

\section{School closure costs}

Our calculations included costs of work absenteeism during school closure events for parents forced to stay home with their healthy children and did not include the hospitalization costs (see Additional file 1). We assumed 2.5 worker days per week loss for the working co-parents and 5 worker days per week loss for working single parents, consistent with values used in other studies [3, 4]. Based on the 2009 Texas census data, we assumed that $20 \%$ of working adults are single parents
[29]. Worker productivity loss due to school closure absenteeism was calculated according to the Human Capital Method, as in [3, 4, 34]: average salary plus benefits times the number of days work lost.

\section{Health outcomes}

For each policy, we determined several health outcomes: number of cases, cases averted by intervention, number of deaths, years of life saved (YLS), total costs, and quality adjusted life-years (QALYs). According to guidelines for program evaluation from the societal perspective [4, 26 , we applied a discount rate of $3 \%$ per annum (range $=1.5 \%$ to $6 \%$ ) to both health and economic outcomes. QALYs were calculated as one of the health outcomes, based on the total years of life saved and the health related quality of life (HRQL) scores for Influenza-Like-Illnesses (ILI) of different age groups considered in this study $[26,27,35]$. The QALY is a measure of health outcome that assigns each period of time a preference-based weight corresponding to the quality of life during that period, i.e., a weight of one corresponds to perfect health and a weight of zero corresponds to a health state equivalent to death [26].

We calculated incremental cost effectiveness ratios (ICER) of each strategy (i.e., the incremental expense incurred for gaining the incremental health outcome when comparing two alternative strategies) as follows:

$$
I C E R=\frac{\text { Total Cost of Strategy }(B)-\text { Total Cost of Strategy }(A)}{\text { Total } Q A L Y(B)-\text { Total } Q A L Y(A)}
$$

We report the ICERs of only the "efficient" closure policies, that is, those that have the greatest positive impact for any level of investment. By convention, all other strategies are considered dominated and were omitted from the cost-effectiveness ratio calculations. (Results for weakly dominated strategies are provided in Tables A6.1-A6.4 in the Additional file 1.) We identified efficient frontiers for each scenario by simultaneously considering the total cost and total QALYs of the interventions.

\section{Sensitivity analyses}

To assess the robustness of our results to uncertainties in critical inputs, we performed deterministic, one-way sensitivity analyses on several critical model parameters: the discounting rate for calculating QALY's lost due to mortality, fraction of working parents that miss work during a school closure, average daily salary, case fatality rates, percent of working parents that are single, and overall employment rate. We varied each parameter from half of its base value to twice its base value, and assessed the impact on the predicted ICER's. 


\section{Results}

We evaluate the epidemiological and cost effectiveness of a spectrum of school closure policies for mitigating an influenza pandemic in the US under four different pandemic scenarios: low transmission-low severity, low transmission-high severity, high transmission-low severity, and high transmission-high severity. In the low transmission scenarios, our model predicts a base case (no intervention) cumulative attack rate (CAR) of $41 \%$ for the school age population and 31\% for the adult population. Analyzing 200 simulations with different samples of $R_{O}$ for each scenario-policy combination, we found that the attack rates tend to decrease with the duration of the closure, and increase with the initial prevalence trigger, that is, with the delay in closing schools (Tables 2 and 3 ).

For the low transmission-low severity and low transmission-high severity scenarios, only the lowest

Table 2 Average Cumulative Attack Rates (CAR) for school age population and adults under the low transmission scenarios (standard deviations in parenthesis)

\begin{tabular}{|c|c|c|c|c|c|c|c|c|}
\hline \multirow{2}{*}{$\begin{array}{l}\text { R0 [1.1-1.5] } \\
\text { Closure } \\
\text { Triggers }\end{array}$} & \multirow[b]{2}{*}{ Populations } & \multicolumn{7}{|c|}{$\begin{array}{l}\text { CAR for different durations (weeks) of } \\
\text { closure for each triggers (\%) }\end{array}$} \\
\hline & & 1 & 2 & 3 & 4 & 8 & 12 & 24 \\
\hline \multirow[t]{4}{*}{$0.50 \%$} & Students & 39.12 & 35.63 & 32.02 & 28.35 & 14.28 & 6.15 & 2.39 \\
\hline & & $(5.8)$ & $(6.9)$ & $(6.3)$ & $(7.3)$ & $(4.9)$ & $(1.9)$ & $(1.7)$ \\
\hline & Adults & 42.45 & 39.30 & 35.88 & 32.30 & 18.52 & 10.52 & 6.88 \\
\hline & & $(4.7)$ & $(5.6)$ & $(5.2)$ & $(6.4)$ & $(4.9)$ & $(2.5)$ & $(2.9)$ \\
\hline \multirow[t]{4}{*}{$0.80 \%$} & Students & 38.34 & 34.67 & 30.88 & 26.93 & 13.60 & 6.81 & 4.02 \\
\hline & & $(6.2)$ & $(6.7)$ & $(7.1)$ & $(6.2)$ & $(4.2)$ & $(1.4)$ & $(1.2)$ \\
\hline & Adults & 41.88 & 38.57 & 35.01 & 31.31 & 18.56 & 12.15 & 9.07 \\
\hline & & $(4.8)$ & (5.6) & $(5.8)$ & $(5.7)$ & $(4.2)$ & $(2.3)$ & $(2.0)$ \\
\hline \multirow[t]{4}{*}{$1.10 \%$} & Students & 37.74 & 33.46 & 29.38 & 25.30 & 13.38 & 8.46 & 6.66 \\
\hline & & $(6.2)$ & $(6.4)$ & $(6.3)$ & $(5.9)$ & $(2.8)$ & $(1.3)$ & $(1.0)$ \\
\hline & Adults & 41.49 & 37.75 & 34.09 & 30.36 & 19.46 & 15.05 & 13.38 \\
\hline & & $(5.1)$ & $(5.3)$ & $(5.5)$ & $(5.2)$ & $(3.2)$ & $(2.8)$ & $(2.2)$ \\
\hline \multirow[t]{4}{*}{$1.40 \%$} & Students & 37.12 & 32.54 & 28.01 & 24.13 & 14.10 & 10.62 & 9.43 \\
\hline & & $(6.2)$ & $(6.0)$ & $(5.9)$ & $(5.4)$ & $(2.0)$ & $(1.9)$ & $(1.8)$ \\
\hline & Adults & 41.08 & 37.18 & 33.27 & 29.89 & 21.06 & 18.14 & 16.90 \\
\hline & & $(5.1)$ & $(4.9)$ & (5.0) & $(4.6)$ & $(2.9)$ & $(3.7)$ & $(3.2)$ \\
\hline \multirow[t]{4}{*}{$1.70 \%$} & Students & 36.69 & 31.63 & 27.32 & 23.63 & 15.58 & 13.27 & 12.40 \\
\hline & & (5.6) & (5.6) & $(5.2)$ & $(4.5)$ & (1.9) & $(2.8)$ & (1.8) \\
\hline & Adults & 40.82 & 36.70 & 33.09 & 29.96 & 23.26 & 21.45 & 20.80 \\
\hline & & $(4.6)$ & $(4.5)$ & $(4.7)$ & (3.9) & $(3.5)$ & $(4.2)$ & $(4.5)$ \\
\hline \multirow[t]{4}{*}{$2.00 \%$} & Students & 36.35 & 31.17 & 27.10 & 24.03 & 17.97 & 16.53 & 16.50 \\
\hline & & $(6.1)$ & $(4.6)$ & $(3.7)$ & $(2.7)$ & (3.3) & $(4.6)$ & $(4.0)$ \\
\hline & Adults & 40.69 & 36.61 & 33.34 & 30.85 & 26.10 & 25.07 & 25.05 \\
\hline & & $(4.8)$ & (3.8) & $(3.5)$ & $(2.8)$ & $(4.6)$ & $(5.5)$ & (5.9) \\
\hline
\end{tabular}

Table 3 Average Cumulative Attack Rates (CAR) for school age population and adults under the high transmission scenarios (standard deviations in parentheses)

\begin{tabular}{lllllllll}
\hline R0 [1.5-2.1] & \multicolumn{7}{c}{$\begin{array}{c}\text { CAR for different durations (weeks) of } \\
\text { closure for }\end{array}$} \\
\hline $\begin{array}{l}\text { Closure } \\
\text { Triggers }\end{array}$ & Populations & 1 & 2 & 3 & 4 & 8 & 12 & 24 \\
$0.50 \%$ & Students & 73.96 & 73.71 & 73.36 & 73.04 & 71.21 & 65.28 & 10.10 \\
& & $(2.7)$ & $(3.3)$ & $(3.3)$ & $(3.5)$ & $(3.7)$ & $(3.1)$ & $(1.4)$ \\
& \multirow{2}{*}{ Adults } & 76.90 & 76.76 & 76.62 & 76.46 & 75.36 & 71.14 & 35.51 \\
& & $(2.2)$ & $(2.2)$ & $(2.1)$ & $(1.9)$ & $(2.1)$ & $(3.8)$ & $(6.0)$
\end{tabular}

0.80\% $\quad$ Students $\quad 73.8073 .28 \quad 72.88 \quad 72.50 \quad 69.8061 .16 \quad 11.40$

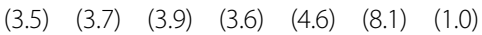

Adults $\quad 76.7876 .6276 .4476 .2474 .67 \quad 68.71 \quad 38.14$

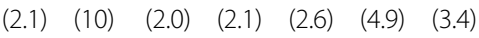

$1.10 \% \quad$ Students $\quad 73.5872 .95 \quad 72.37 \quad 71.85 \quad 68.31 \quad 56.65 \quad 12.90$ $\begin{array}{lllllll}(3.8) & \text { (3.3) } & \text { (3.6) } & \text { (3.2) } & (4.8) & (7.9) & (1.0)\end{array}$

Adults $\quad 76.7476 .4776 .2175 .9473 .8866 .1640 .34$ $\begin{array}{llllll}(2.2) & (2.1) & (2.2) & (2.3) & (2.6) & (4.4)\end{array}$

$1.40 \% \quad$ Students $\quad 73.3272 .5171 .90 \quad 71.1366 .54 \quad 51.80 \quad 14.55$

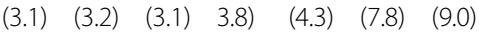

Adults $\quad 76.6176 .3075 .9775 .6673 .02 \quad 63.52 \quad 42.44$

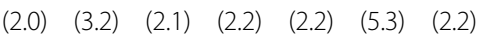

$1.70 \% \quad$ Students $\quad 73.22 \quad 72.15 \quad 71.28 \quad 70.47 \quad 64.81 \quad 47.38 \quad 15.90$

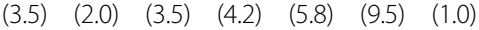

Adults $\quad 76.5876 .1375 .7475 .3572 .1361 .2644 .02$

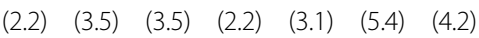

$2.00 \% \quad$ Students $\quad 73.00 \quad 71.80 \quad 70.74 \quad 69.62 \quad 62.98 \quad 43.48 \quad 17.30$ $\begin{array}{lllllll}(3.4) & (2.2) & (2.0) & (3.9) & (5.7) & (9.4) & (1.5)\end{array}$

Adults $\quad 76.5275 .9675 .5174 .9971 .2259 .2545 .15$

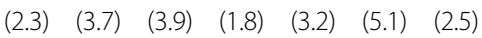
3\% $\quad$ Students $\quad 72.28 \quad 70.4968 .75 \quad 67.21 \quad 56.47 \quad 31.82 \quad 21.80$ $\begin{array}{lllllll}(3.4) & (1.9) & (2.0) & (3.9) & (7.1) & (4.8) & (2.0)\end{array}$ Adults $\quad 53.33 \quad 52.8052 .2451 .73 \quad 47.5939 .04 \quad 49.40$

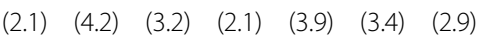
4\% $\quad$ Students $\quad 71.56 \quad 69.0466 .8064 .47 \quad 50.04 \quad 32.20 \quad 26.60$ $\begin{array}{lllllll}(3.4) & (3.5) & (2.1) & (4.2) & (7.6) & (2.7) & (2.0)\end{array}$ Adults $\quad 53.1252 .3951 .7050 .91 \quad 45.47 \quad 38.92 \quad 52.95$

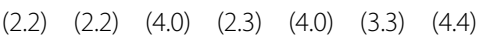
5\% $\quad$ Students $\quad 70.8767 .6364 .6861 .61 \quad 45.40 \quad 33.34 \quad 30.40$

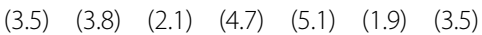
Adults $\quad 52.94 \quad 51.97 \quad 51.04 \quad 50.03 \quad 44.13 \quad 39.98 \quad 55.90$ $\begin{array}{lllllll}(2.2) & (2.2) & (4.5) & (2.5) & (3.2) & (3.4) & (4.0)\end{array}$ 6\% Students $70.16 \quad 66.24 \quad 62.58 \quad 58.91 \quad 43.41 \quad 36.35 \quad 35.30$ $\begin{array}{lllllll}(3.8) & (3.7) & (4.1) & (5.0) & (3.1) & (3.0) & (4.0)\end{array}$ Adults

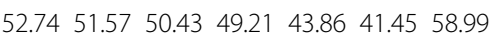
$\begin{array}{lllllll}(2.3) & (2.3) & (2.7) & (3.1) & (3.1) & \text { (3.3) } & \text { (4.0) }\end{array}$ 

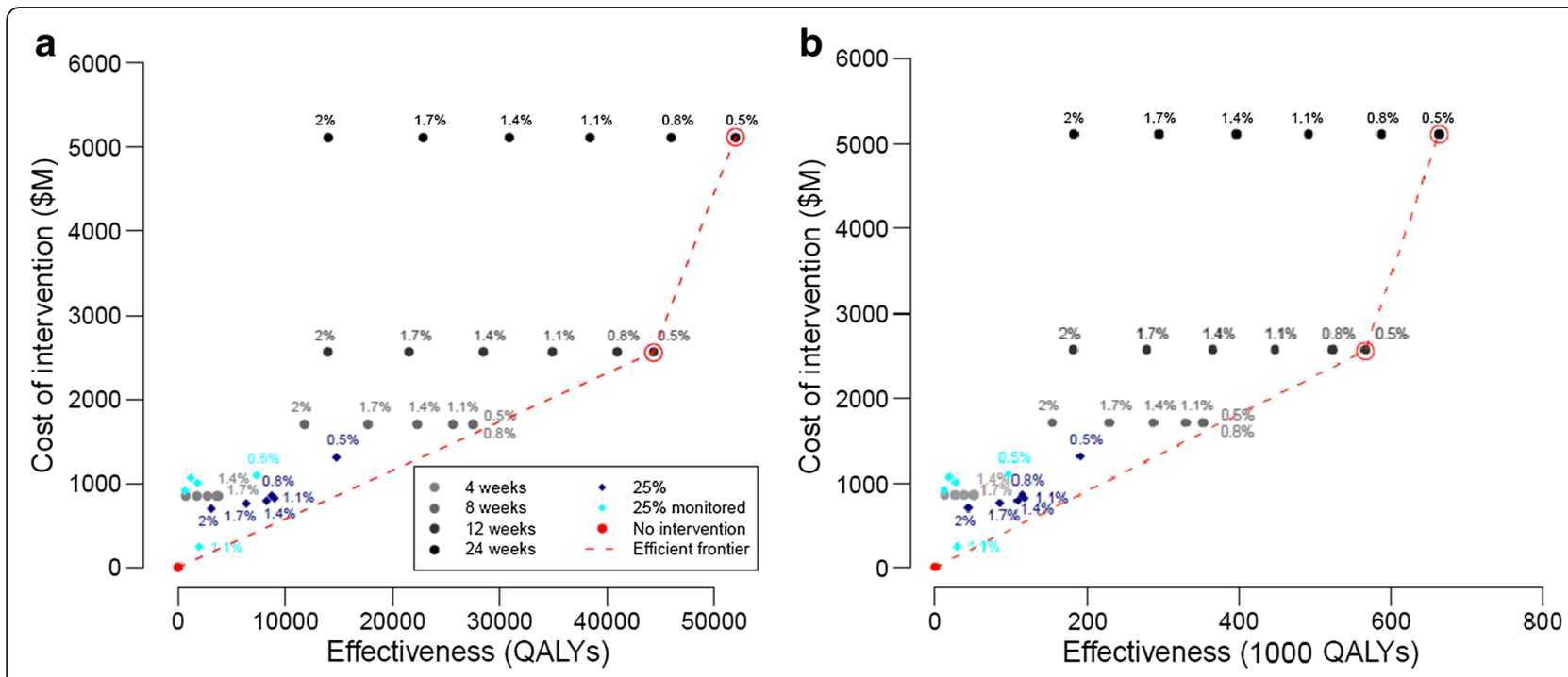

Figure 2 a: Cost and effectiveness comparison of school closure strategies with different closure triggers for low transmission-low CFR scenario. Red circles indicate efficient strategies. b: Cost and effectiveness comparison of school closure strategies with different closure triggers for low transmission-high CFR scenario. Red circles indicate efficient strategies.

trigger value yields efficient strategies: a $0.5 \%$ SAP closure trigger followed by either a 12 -week closure $(\$ 2.56$ billion societal cost and total of 44,300 QALYs) or a 24week closure ( $\$ 5.12$ billion societal cost and 51,900 QALYs) (Figure 2a and b). For the 12-week closure policy, as the severity increases from a case fatality rate of less than $0.2 \%$ to $2 \%$, the incremental cost effectiveness ratio (ICER), that is, the cost per QALY gain, decreases from $\$ 57,700$ to $\$ 4,500$ and the expected number of deaths averted rises from 4,250 to 84,188 (however, the proportion of deaths averted increases more modestly from 0.67 to 0.73 ). For a 24-week closure policy, as the severity increases, the ICER decreases from $\$ 334,800$ to $\$ 26,400$ and the expected number of deaths averted increase from 4,981 to 96,702 (the proportion of deaths averted increases from 0.79 to 0.84 ) (Tables 4 and 5).

For the high transmission scenarios, our model predicts that $72 \%$ of the school age population and $52 \%$ of the adult population will become infected. Based on an evaluation of total costs and total QALYs for each school closure strategy, three efficient strategies emerged for the high transmission-low severity scenario (Figure 3a): (i) a 1.1\%

Table 4 Incremental Cost Effectiveness Ratio (ICER) of the effective closure strategies under the low transmission-low severity scenario

\begin{tabular}{llllll}
\hline Intervention & $\begin{array}{l}\text { Total } \\
\text { Deaths }\end{array}$ & $\begin{array}{l}\text { YOL } \\
\text { Saved }\end{array}$ & $\begin{array}{l}\text { Total } \\
\text { QALYs } \\
\text { Gained }\end{array}$ & $\begin{array}{l}\text { Total } \\
\text { Closure } \\
\text { Cost } \mathbf{( \$ )}\end{array}$ & $\begin{array}{l}\text { ICER } \\
\text { (\$/QALY) }\end{array}$ \\
\hline No closure & 6,340 & - & & 0.00 & - \\
$0.5 \%, 12 \mathrm{~W}$ & 2,090 & $133,612.88$ & 44,300 & $2,560,372,219$ & 57,700 \\
$0.5 \%, 24 \mathrm{~W}$ & 1,359 & $156,209.94$ & 51,900 & $5,120,744,439$ & 334,800 \\
\hline
\end{tabular}

closure trigger coupled with a non-monitored prevalencebased reopening trigger (specifically, a decrease in SAP to $25 \%$ of the original value, that is, a decrease in SAP from $1.1 \%$ to $0.275 \%$ ), (ii) a $0.5 \%$ closure trigger with nonmonitored prevalence-based reopening trigger $(50 \%$ decrease in the SAP from the closure trigger), and (iii) a $0.5 \%$ closure trigger followed by 24 weeks of closure. Under the first of these policies, the model predicts that schools will close for an average of 125 days (standard deviation 3.67 days) while prevalence declines to the re-opening threshold (Figure 4). Thus, the closure duration falls between the fixed closure options of 12 and 24 weeks. A non-monitored (NM) policy assumes that accurate estimates of SAP are made throughout the closure period with a one-week lag. For the high transmission-high severity, two additional efficient strategies exist; these are 3\% closure trigger with non-monitored prevalence-based reopening until SAP drops to $25 \%$ of the trigger and $0.8 \%$ closure trigger with monitoring $(\mathrm{M})$ based reopening until SAP drops to $25 \%$ of the original trigger value (See Figure 3b).

As in the low transmission scenarios, the efficient policies save more lives and have considerably lower ICERs

Table 5 Incremental Cost Effectiveness Ratio (ICER) of the effective closure strategies under the low transmission-high severity scenario

\begin{tabular}{llllll}
\hline Intervention & $\begin{array}{l}\text { Total } \\
\text { Deaths }\end{array}$ & $\begin{array}{l}\text { YOL } \\
\text { Saved }\end{array}$ & $\begin{array}{l}\text { Total } \\
\text { QALYs } \\
\text { Gained }\end{array}$ & $\begin{array}{l}\text { Total } \\
\text { Closure } \\
\text { Cost } \mathbf{( \$ )}\end{array}$ & $\begin{array}{l}\text { ICER } \\
\text { (\$/QALY) }\end{array}$ \\
\hline No closure & 115,780 & - & - & 0.00 & - \\
$0.5 \%, 12 \mathrm{~W}$ & 31,592 & $3,748,812.28$ & 566,500 & $2,560,372,219$ & 4,500 \\
$0.5 \%, 24 \mathrm{~W}$ & 19,078 & $4,256,198.65$ & 663,600 & $5,120,744,439$ & 26,400 \\
\hline
\end{tabular}



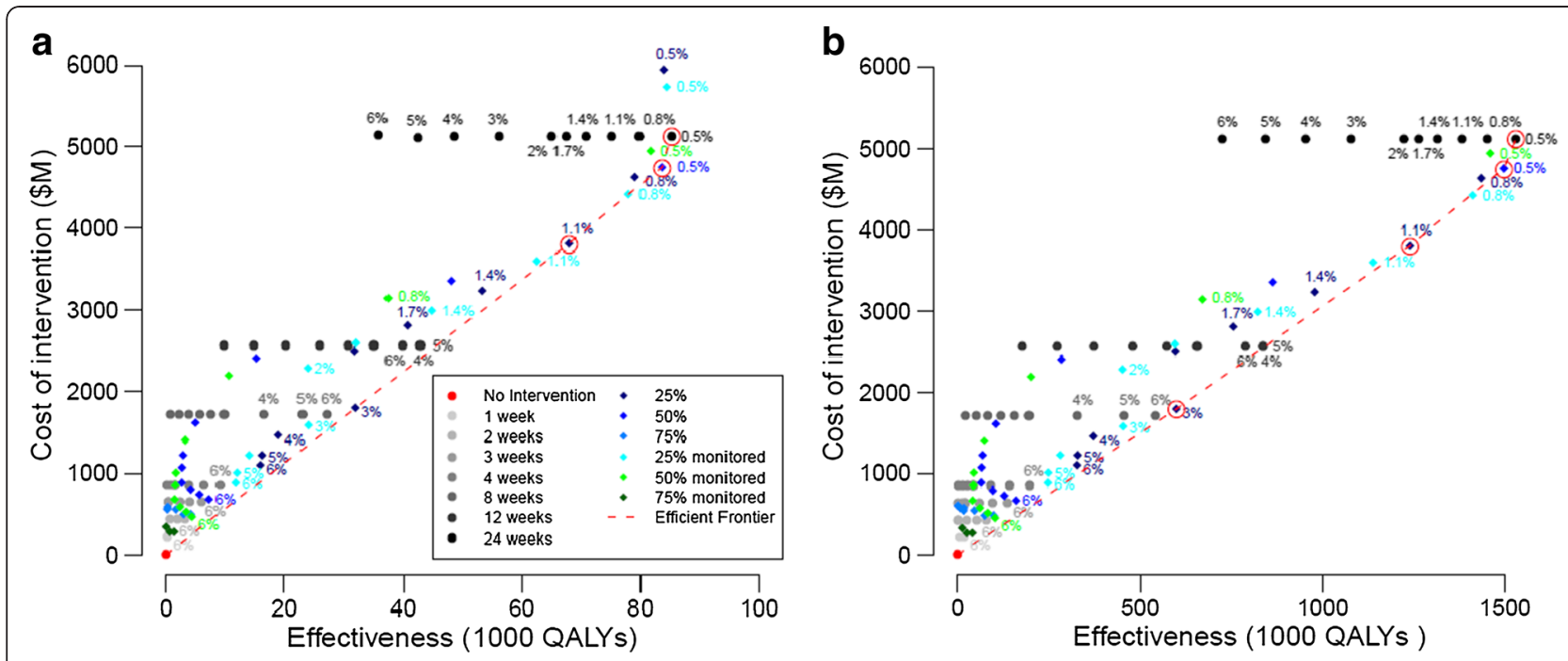

Figure 3 a: Cost and effectiveness comparison of school closure strategies with different closure triggers for high transmission-low CFR scenario. Red circles indicate efficient strategies. b: Cost and effectiveness comparison of school closure strategies with different closure triggers for high transmission-high CFR scenario. Red circles indicate efficient strategies.

under the high severity scenario than under the low severity scenario (Tables 6 and 7). For example, for the $1.1 \%$ SAP trigger coupled with reopening after a reduction in SAP to $25 \%$ of its original value (1.1\%, NM25\%), the incremental cost per QALY gained decreases from $\$ 56,000$ to $\$ 3,100$ and the number of deaths averted with school closure increases from 4,081 to 187,307 (the proportion of deaths averted increases from 0.27 to 0.42).

Our analyses suggest that efficient strategies depend on the transmission rate of the strain (Tables 3, 4, 5, 6, 7). Although there is some overlap in the efficient sets for the low and high transmission rate scenarios, there are also notable differences. For a relatively slowly spreading strain, an early implementation and relatively long closure $(0.5 \%, 12 \mathrm{w}$ or $0.5 \%, 24 \mathrm{w})$ is efficient. For more rapidly spreading strains, later closures (prevalence thresholds ranging from $0.5 \%$ to $3 \%$ ) are viable, but require variable durations. Specifically, the $3 \%, \mathrm{NM} 25 \%$; $1.1 \%, \mathrm{NM} 25 \% ; 0.8 \%, \mathrm{M} 25 \%$; and $0.5 \%, \mathrm{NM} 50 \%$ strategies are predicted to last $59,125,145$, and 155 days, respectively (with standard deviations of 2.50, 3.28, 3.50, and 4.12 respectively). The ICERs of the efficient strategies are generally predicted to be lower with higher transmissibility and higher severity (Tables 4, 5, 6, 7).

The World Health Organization (WHO) suggests that health interventions be designated cost-effective if they deliver QALYs at a cost less than three times a nation's per capita GDP and very cost-effective if the cost per QALY is less than the country's per capita GDP [36]. To assess the cost effectiveness of school closure policies, we considered closure durations relative to the US per capita GDP in 2009 (approximately \$46,000 according to [36]). Our analyses show that for the low transmission-low

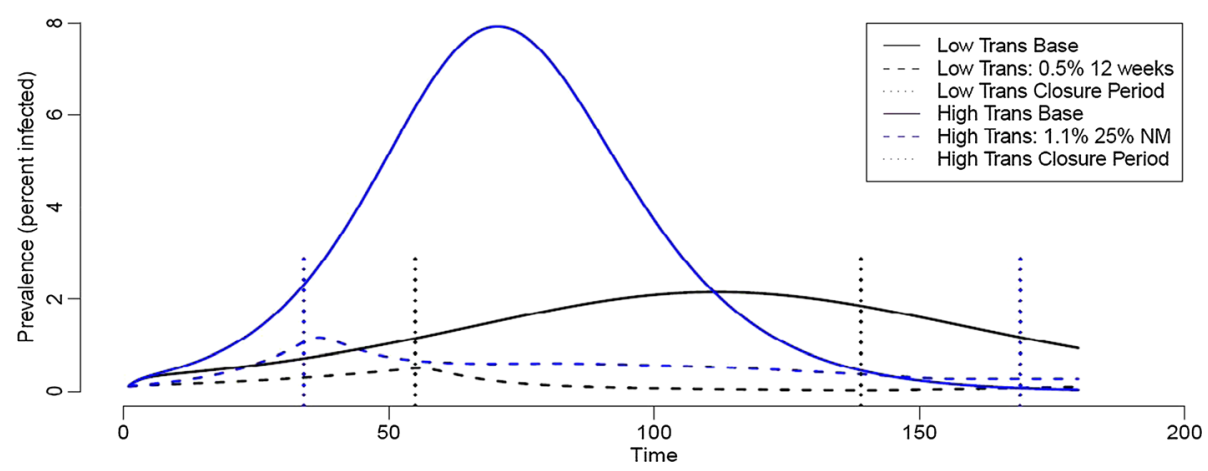

Figure 4 Total influenza prevalence curves with and without school closures under low transmission (black) and high transmission (blue) scenarios. Dashed lines show a typical epidemic curve under a cost effective closure policy (based on one simulation). Vertical dotted lines indicate beginning and end of each school closure. 
Table 6 Incremental Cost Effectiveness Ratio (ICER) of the effective closure strategies under the high transmissionlow severity scenario

\begin{tabular}{llllll}
\hline Intervention & $\begin{array}{l}\text { Total } \\
\text { Deaths }\end{array}$ & $\begin{array}{l}\text { YOL } \\
\text { Saved }\end{array}$ & $\begin{array}{l}\text { Total } \\
\text { QALYs } \\
\text { Gained }\end{array}$ & $\begin{array}{l}\text { Total } \\
\text { Closure } \\
\text { Cost } \mathbf{( \$ )}\end{array}$ & $\begin{array}{l}\text { ICER } \\
\text { (\$/QALY) }\end{array}$ \\
\hline No closure & 15,182 & - & 0.00 & 0.00 & - \\
$1.1 \%, N M 25 \%$ & 11,101 & $129,858.17$ & 67,900 & $3,810,077,708$ & 56,100 \\
$0.5 \%, N M 50 \%$ & 8,667 & $204,696.57$ & 83,600 & $4,754,976,979$ & 60,200 \\
$0.5 \%, 24 \mathrm{~W}$ & 7,167 & $251,156.96$ & 85,200 & $5,120,744,439$ & 223,800 \\
\hline
\end{tabular}

severity scenario, a $0.5 \%$ prevalence closure trigger followed by a 12 -week closure ( $\$ 57,700$ per QALY gain) is the only effective and cost effective strategy (i.e., its ICER is less than three times the US per capita GDP). For the low transmission-high severity scenario, a $0.5 \%$ prevalence closure trigger followed by either a 12-week closure $(\$ 4,500$ per QALY gain) or 24-week closure $(\$ 26,300$ per QALY gain) meets the very cost effective threshold. Thus, the $5 \%, 24 \mathrm{w}$ would be the preferred policy, as it is the largest one (conferring the greatest number of QALYs) with a cost-effectiveness ratio below the willingness-to-pay threshold.

For the high transmission-low severity scenario, a $1.1 \%$ closure trigger coupled with a non-monitored prevalence-based reopening trigger (a decrease in SAP to $0.275 \%$ ), and a $0.5 \%$ closure trigger with nonmonitored prevalence-based reopening trigger $(50 \%$ decrease in the SAP to $0.25 \%$ ) are cost effective but not very cost effective, with costs of $\$ 56,000$ per QALY gain and $\$ 60,200$ per QALY gain, respectively. The latter of the two is larger (higher total QALY's gained), and thus would be the preferred choice. Finally, for the high transmission-high severity scenario, all five efficient strategies meet the very cost effective threshold, with the 0.5\% SAP trigger, 24-week closure being the preferred program. Our cost estimates are comparable to published values (Table 8). For the low transmission-low severity scenario, influenza is predicted to cost the State of Texas $0.023-0.5 \%$ of its GDP.

Table 7 Incremental Cost Effectiveness Ratio (ICER) of the effective closure strategies under the high transmissionhigh severity scenario

\begin{tabular}{llllll}
\hline Intervention & $\begin{array}{l}\text { Total } \\
\text { Deaths }\end{array}$ & $\begin{array}{l}\text { YOL } \\
\text { Saved }\end{array}$ & $\begin{array}{l}\text { Total } \\
\text { QALYs } \\
\text { Gained }\end{array}$ & $\begin{array}{l}\text { Total } \\
\text { Closure } \\
\text { Cost (\$) }\end{array}$ & $\begin{array}{l}\text { ICER } \\
\text { (\$/QALY) }\end{array}$ \\
\hline No closure & 444,157 & - & 0 & 0.00 & - \\
$3 \%, N M 25 \%$ & 255,762 & $6,577,339.87$ & 599,300 & $1,798,356,678$ & 3,000 \\
$1.1 \%, N M 25 \%$ & 256,850 & $6,524,695.19$ & $1,239,800$ & $3,810,077,708$ & 3,100 \\
$0.8 \%, M 25 \%$ & 255,517 & $6,587,498.52$ & $1,411,800$ & $4,419,690,141$ & 3,500 \\
$0.5 \%, N M 50 \%$ & 250,337 & $6,660,573.77$ & $1,497,300$ & $4,754,976,979$ & 3,900 \\
$0.5 \%, 24 W$ & 249,206 & $6,889,044.94$ & $1,529,600$ & $5,120,744,439$ & 11,300 \\
\hline
\end{tabular}

For both high and low transmission pandemics, the predicted ICER values varied considerably with the percentage of working adults who will miss work due to influenza, average daily salary, and case fatality rate (CFR). For example, we illustrate this with a tornado diagram for the preferred strategy under the high transmission-high severity pandemic scenario (Figure 5). Increases in any of the parameters, except the CFR, lead to increases in the ICER of the school closure policies. Conversely, decreases in pandemic severity (CFR) lead to increases in ICER of the closure policy. None of these parameter perturbations cause the policy to lose its very cost effective designation, that is, the ICER remains well below the willingness-topay threshold of $\$ 46,000$. The parameters influence the ICER values similarly for the cost effective policies under the other three pandemic influenza scenarios (Figures A4.1-A4.3 in Additional file 1). While none of the perturbations cause the preferred policies rise above the cost effectiveness willingness-to-pay threshold, some extreme parameter perturbations are predicted to move policies from very cost effective to cost effective (by increasing the ICER) or, vice versa, from cost effective to very cost effective (by reducing the ICER).

\section{Discussion and conclusions}

School closure interventions, in general social distancing interventions and population behavior changes, will lead to a delayed impact of the overall pandemic by reducing the peak of the current pandemic wave and overall epidemic size. However, it is expected that the temporarily spared susceptible population during the first wave can be affected during subsequent second or third pandemic waves. It is also important to note that it may take number of weeks before reliable estimates of pandemic severity could be inferred and this could impact the effectiveness of policy decisions on whether school closures should be triggered. This was the case during the 2009 A/H1N1 pandemic in Mexico; when the Mexican government decided to close schools across the country based on the available information, there was no reliable estimate of the case fatality ratio.

According to our model, school closures can significantly reduce the total number of influenza cases, but the epidemiological impact and societal costs of a school closure critically depend on the timing and duration of the closure. In the early days of a pandemic, there is typically considerable uncertainty about its transmission rate (or $R_{0}$ ) and its severity (or CFR). Policy makers face two interrelated decisions: (1) whether or not to implement a school closure policy; and (2) if so, which one. The answer to the first question depends on the predicted effectiveness and cost effectiveness of the effective policy options. We find that relatively few closure policies are efficient under the low transmissibility scenarios, while several are 
Table 8 Comparison to published analyses

\begin{tabular}{llllll}
\hline & Cost of Influenza & Cost of Closure & Closure Duration & Population & \$ Amount \\
\hline Sadique et al. & - & $0.2-1 \%$ GDP & 12 weeks & England & $0.2-1.2$ Billion Sterlin \\
Lempel et al. & - & $0.1-0.3 \%$ GDP & 4 weeks & US & $10-47$ Billion \$ \\
Smith et al. & $3.3-4.3 \%$ GDP & - & 4 weeks & UK & $85.8-97.6$ Billion Sterling \\
Brouwers et al. & $2-3 \%$ GDP & - & - & Sweden & 2.5 Billion SEK \\
Sander et al. & - & - & 26 weeks & US & 2720 \$/person \\
Araz et al. & & $0.023-0.5 \%$ GDP & $1-24$ weeks & Texas & $0.2-5.12$ Billion \$ \\
\hline
\end{tabular}

efficient under the high transmissibility scenarios. The answer to the second question depends on both the transmission rate and severity of the pandemic. Slower spreading pandemics call for early triggers and relatively long duration closures (12 weeks and 24 weeks), regardless of severity; and more rapidly spreading pandemics allow for higher triggers (e.g. 1.1\% and 3\%) coupled with moderate durations (averages of 125 days and 59 days, respectively), depending on the severity of the strain (see Additional file 1 for timing of the triggers for both cases). Although we assumed that the SAP estimates are exact in the model and single step errors, e.g. a policy intending to close school at a SAP of $0.8 \%$ actually closing school at $0.5 \%$ or $1.1 \%$, do not make large differences in CARs, it is worth mentioning that these kind of errors can generate dramatic switches in the effectiveness of policy implementation, e.g. a switch from a cost effective strategy to a dominated or weakly dominated strategy (e.g. see low transmission, low CFR scenario in the Additional file 1 Table A6.1).

The policy options included fixed duration closures and re-opening triggers based on decreases in schoolaged disease prevalence, both with and without additional surveillance to improve real-time prevalence estimates. Our model assumed that, in the absence of monitoring, decision-makers receive accurate estimates of prevalence with a one-week delay, and monitoring simply removes the delay. Although the direct cost of monitoring is assumed to be low, most of the efficient policies are either fixed duration or have non-monitored reopening triggers. This suggests that slightly lower reopening thresholds than those considered may provide a better balance between costs of closure and health outcome. Similarly, the efficiency of several trigger-based re-opening policies may indicate that there are better fixed durations than the values considered.

In summary, we have integrated a mathematical model of influenza transmission dynamics into a costeffectiveness analytic framework for evaluating a wide range of school closure and reopening policies with respect to their societal costs and health impacts. The presented rigorous approach of this paper can be adapted to evaluate and compare a variety of non-pharmaceutical , vaccine, and antiviral policy options for influenza. We have found that the transmission rate and case fatality rate of a spreading pandemic can dramatically impact

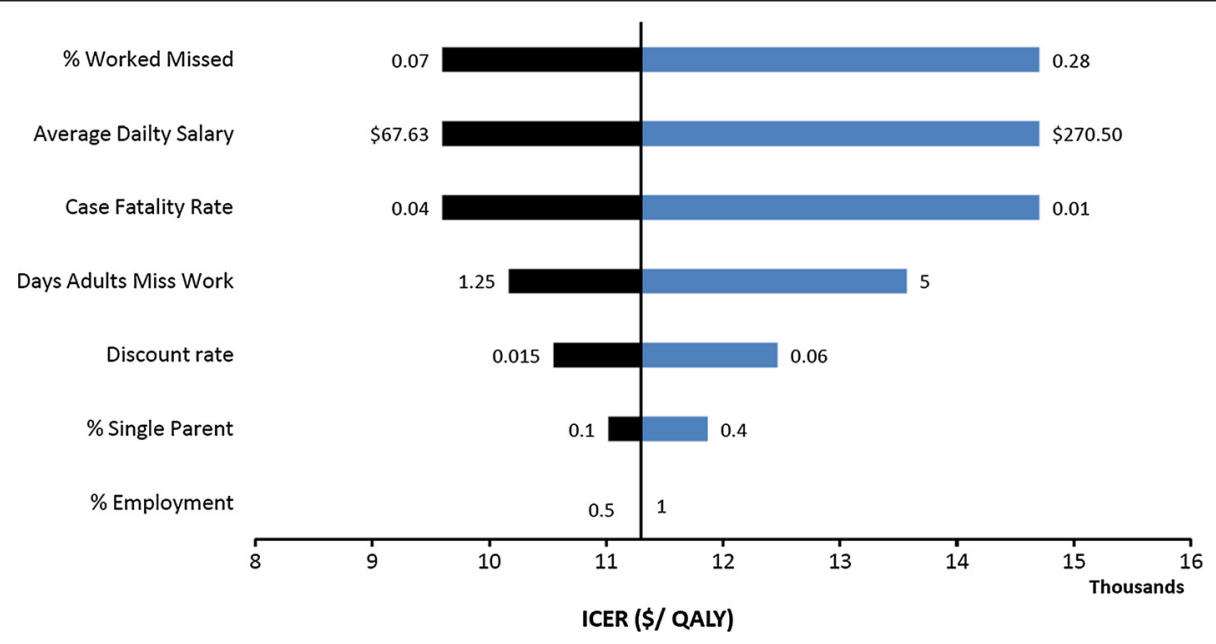

Figure 5 Tornado diagram comparing the relative impact of input variables on the ICER for the preferred closure policy $(0.5 \%$ SAP trigger, 24-week) under the High transmission-High severity scenario. The width of the bars indicates the uncertainty associated with each parameter as it ranges from $50 \%$ of its base value to two times of its base value, as given in Additional file 1. 
whether or not a school closure policy is efficient and cost effective. Although not surprising, this highlights the importance of obtaining early and reliable estimates of pandemic severity to public health decision-making.

\section{Additional file}

Additional file 1: Appendix 1-6 [37]

\section{Competing interests}

The authors declare that they have no competing interest.

\section{Authors' contribution}

In this study, OA conducted the majority of the analyses and was the principal author of the manuscript. LM and PD designed the study and directed its implementation, including quality assurance and control, and preparation of the manuscript. AG and DP provided critical input into the analytic techniques and preparation of the manuscript. SB and BG contributed to the implementation of the models and analysis of the model results. All authors read and approved the final manuscript.

\section{Acknowledgment}

This work was supported by the Models of Infectious Disease Agent Study (MIDAS) of the National Institute of General Medical Sciences (NIGMS) at the National Institutes of Health (NIH) [U01 $\mathrm{g}$ to LAM and APG].

\section{Author details}

${ }^{1}$ College of Public Health, University of Nebraska Medical Center, Omaha, NE 68198, USA. ${ }^{2}$ Information Risk and Operations Management Department, McCombs School of Business, The University of Texas, Austin, TX 78712, USA. ${ }^{3}$ School of Medicine, Yale University, CT, New Haven 06510, USA. ${ }^{4}$ Section of Integrative Biology, The University of Texas at Austin, Austin, TX 78712, USA. ${ }^{5}$ Epidemiology and Public Health, New Haven, CT 06510, USA. 'Santa Fe Institute, Santa Fe, NM, USA.

Received: 13 December 2011 Accepted: 7 May 2012

Published: 18 June 2012

\section{References}

1. Fraser C, Donnelly CA, Cauchemez S, Hanage WP, Van Kerkhove MD, Hollingsworth TD, Griffin J, Baggaley RF, Jenkins HE, Lyons EJ, Jombart T, Hinsley WR, Grassly NC, Balloux F, Ghani AC, Ferguson NM, Rambaut A, Pybus OG, Lopez-Gatell H, Alpuche-Aranda CM, Chapela I, Zavala EP, Guevara DME, Checchi F, Garcia E, Hugonnet S, Roth C, The WHO: Collaboration Rapid Pandemic Assessment: Pandemic Potential of a Strain of Influenza A (H1N1): Early Findings. Science 2009, 234:1557.

2. Nishiura H, Chowell G, Safa M, Castillo-Chavez C: Pros and cons of estimating the reproduction number from early epidemic growth rate of influenza A (H1N1) 2009. Theoretical biology and Medical Modelling 2010, 7:1

3. Lempel $\mathrm{H}$, Hammond RA, Epstein JM: Economic cost and health care workforce effects of school closures in the US. PLoS Curr Influenza 2009, 5: RRN1051.

4. Sander B, MecDev MBA, Nizam A, Garisson LP, Postma MJ, Halloran ME, Longini IM: Economic Evaluation of Influenza Pandemic Mitigation Strategies in the United States Using a Stochastic Microsimulation transmission model. Value in Health 2009, 12:226-233.

5. Halder N, Kelso JK, Milne GJ: Developing guidelines for school closure interventions to be used during a future influenza pandemic. BMC Infect Dis 2010, 10:221

6. Lee BY, Brown ST, Cooley P, Potter MA, Wheaton WD, Voorhees RE, Stebbins S, Grefenstette JJ, Zimmer SM, Zimmerman RK, Assi TM, Bailey RR, Wagener DK, Burke DS: Simulating school closure strategies to mitigate an influenza epidemic. J Public Health Manag Pract. 2010, 16(3):252-61.

7. Sasaki A, Gatewood A, Ozonoff A, Suzuki H, Tanabe N, Seki N, Saito R, Brownstein JS: Evidence-based tool for triggering school closures during influenza outbreaks, Japan. Emerging Infect Dis 2009, 15:1841-3.
8. Cauchemez S, Valleron AJ, Boelle PY, Flahault A, Ferguson NM: Estimating the impact of school closure on influenza transmission from Sentinel data. Nature 2008, 452:750-754. PubMed DOI DOI:dx.doi.org.

9. Ferguson NM, Cummings DA, Cauchemez S, Fraser C, Riley S, Meeyai A, lamsirithaworn S, Burke DS: Strategies for containing an emerging influenza pandemic in Southeast Asia. Nature 2005, 437:209-214.

10. Hens N, Ayele GM, Goeyvaerts N, Aerts M, Mossong J, Edmunds JW, Beutels $P$ : Estimating the impact of school closure on social mixing behavior and the transmission of close contact infections in eight European countries. BMC Infect Dis 2009, 9:187.

11. Milne GJ, Kels JK, Kelly HA, Huband ST, McVernon J: A Small Community Model for the Transmission of Infectious Diseases: Comparison of School Closure as an Intervention in Individual-Based Models of an Influenza Pandemic. PLoS One 2008, 3(12):e4005. doi:10.1371/journal.pone.0004005.

12. Gojovic MZ, Sande B, Fisman D, Krahn MD, Bauch CT: Modeling mitigation strategies for pandemic (H1N1). CMAJ 2009, 181(10):673-677.

13. Kelso JK, Milne GJ, Kelly H: Simulation suggests that rapid activation of social distancing can arrest epidemic development due to a novel strain of influenza. BMC Publ Health 2009, 9:117.

14. Brouwers L, Cakici B, Camitz M, Tegnell A, Boman M: Economic consequences to society of pandemic H1N1 influenza 2009 - preliminary results for Sweden. Euro Surveill 2009, 14(37)

15. Fitzner KA, Shortridge KF, MCGhee SM, Hedley AJ: Cost-effectiveness study on influenza prevention in Hong-Kong. Health Policy 2001, 56:215-234.

16. Keogh-Brown MR, Wren-Lewis S, Edmunds WJ: Beutels P. Smith RD: Calculating the macroeconomic effects on the UK of an influenza pandemic. Heal Econ; 2009.

17. Sadique MZ, Adams EJ, Edmunds WJ: Estimating the costs of school closure for mitigating an influenza pandemic. BMC Publ Health 2008, 8:15.

18. Smith RD, Keogh-Brown MR, Barnett T, Tait J: The economy-wide impact of pandemic influenza on the UK: a computable general equilibrium modelling experiment. BMJ 2009, 339:b4571.

19. Halder N, Kelso JK, Milne GJ: Cost effective strategies for mitigating a future influenza pandemic with H1N1 2009 characteristics. PLoS One 2011, 6(7):e22087. doi:10.1371/journal.pone0022087.

20. Khazeni N, Hutton DW, Garber AM, Owens DK: Effectiveness and cost effectiveness of expanded antiviral prophylaxis and adjuvanted vaccination strategies for influenza A (H5N1). Ann Intern Med 2009, 151:840-853.

21. Lee VJ, Tok MY, Chow VT, Phua KH, Ooi EE, Tambyah PA, Chen MI: Economic analysis of pandemic influenza vaccination strategies in Singapore. PLoS One 2009, 4(9).

22. Sander B, Kwing JC, Bauch CT, Maetzel A, McGeer A, Raboud JM, Krahn M: Economic appraisal of Ontario's universal influenza immunization program: cost-utility analysis. Plos Med 2010, 7(4):e1000256. doi:10.1371/ journal.pmed.1000256.

23. Perlroth DJ, Glass RJ, Davey VJ, Cannon D, Garber AM, Owens DK: Health outcomes and cost of community mitigation strategies for an influenza pandemic in the United States. Clin Infect Dis 2010, 50(2):165-174.

24. Cauchemez S, Ferguson NM, Wachtel C, Tegnell A, Saour G, Duncan B, Nicoll A: Closure of school during an influenza pandemic. Lancet Infect Dis 2009, 9(8):473-481.

25. Dimitrov NB, Meyers LA: Mathematical Approaches to Infectious Disease Prediction and Control. J. J. Hasenbein, ed. INFORMS TutORials in Operations Research 2010, 7:1-25.

26. Gold MR, Siegel JE, Russel LB, Weinstein MC: Cost-Effectiveness in Health and Medicine. Report of the Panel on Cost-effectiveness in Health and Medicine. New York: Oxford University Press; 1996.

27. Muenning P: Designing and conducting cost-effectiveness analyses in medicine and healthcare. San Francisco: John Wiley \& Sons, Inc; 2002.

28. Anderson RM, May RM: Infectious Diseases of Humans: Dynamics and Control. New York: Oxford University Press; 1991

29. FedStat, Federal Statistical Agency: 2009, http://fedstats.gov/(Accessed in January, 2010.

30. Mossong J, Hens $\mathrm{N}$, Jit M: Social contacts and mixing patterns relevant to the spread of infectious diseases. PLoS Medicine 2008, 5(3):e74

31. Presanis AM, De Angelis D, Hagy A, Reed C, Riley S, et al: The Severity of Pandemic H1N1 Influenza in the United States, from April to July 2009: A Bayesian Analysis. PLoS Med 2009, 6(12):e1000207. doi:10.1371/journal. pmed. 1000207. 
32. Chowell G, Ammon CE, Hengartne NW, Hyman JM: Transmission dynamics of the great influenza pandemic of 1918 in Geneva, Switzerland: Assessing the effects of hypothetical interventions. J Theor Biol 2006, 24 (2):193-204.

33. Pourbohloul B, Ahued A, Davoudi B, Meza R, Meyers LA, Skowronski DM, Villaseñor I, Galván F, Cravioto P, Earn DJD, Dushoff J, Fisman D, Edmunds WJ, Hupert N, Scarpino SV, Trujillo J, Lutzow M, Morales J, Contreras A, Chávez C, Patrick DM, Brunham RC: Initial human transmission dynamics of the pandemic (H1N1) 2009 virus in North America. Influenza and Other Respiratory Viruses 2009, 3:215-222.

34. Hak E, Meijboom MJ, Buskens E: Modelling the health-economic impact of next influenza pandemic in The Netherlands. Vaccine 2006, 24:6756-6760.

35. Turner D, Wailoo A, Nicholon K, Cooper N, Sutton A, et al: Systematic review and economic decision modelling for the prevention and treatment of influenza A and B. Health Technology Assess 2003, 7:1-170.

36. WHO: Cost effectiveness thresholds. 2009,

37. Keeling MJ, Rohani P: Modeling infectious diseases in humans and animals. New Jersey: Princeton University Press; 2008.

doi:10.1186/1471-2458-12-449

Cite this article as: Araz et al:: Simulating school closure policies for cost

effective pandemic decision making. BMC Public Health 2012 12:449.

\section{Submit your next manuscript to BioMed Central and take full advantage of:}

- Convenient online submission

- Thorough peer review

- No space constraints or color figure charges

- Immediate publication on acceptance

- Inclusion in PubMed, CAS, Scopus and Google Scholar

- Research which is freely available for redistribution 